\title{
Beyond the Bakushinkii veto: regularising linear inverse problems without knowing the noise distribution
}

\author{
Bastian Harrach ${ }^{1} \cdot$ Tim Jahn $^{1} \cdot$ Roland Potthast ${ }^{2}$ \\ Received: 15 November 2018 / Revised: 25 March 2020 / Published online: 26 May 2020 \\ (c) The Author(s) 2020
}

\begin{abstract}
This article deals with the solution of linear ill-posed equations in Hilbert spaces. Often, one only has a corrupted measurement of the right hand side at hand and the Bakushinskii veto tells us, that we are not able to solve the equation if we do not know the noise level. But in applications it is ad hoc unrealistic to know the error of a measurement. In practice, the error of a measurement may often be estimated through averaging of multiple measurements. We integrated that in our anlaysis and obtained convergence to the true solution, with the only assumption that the measurements are unbiased, independent and identically distributed according to an unknown distribution.
\end{abstract}

Mathematics Subject Classification 65J22 (Numerical solution to inverse problems in abstract spaces)

\section{Introduction}

The goal is to solve the ill-posed equation $K \hat{x}=\hat{y}$, where $\hat{x} \in \mathscr{X}$ and $\hat{y} \in \mathscr{Y}$ are elements of infinite dimensional Hilbert spaces and $K$ is either linear and bounded with non-closed range, or more specifically compact. We do not know the right hand side $\hat{y}$ exactly, but we are given several measurements $Y_{1}, Y_{2}, \ldots$ of it, which are independent, identically distributed and unbiased $\left(\mathbb{E} Y_{i}=\hat{y}\right)$ random variables. Thus we assume, that we are able to measure the right hand side multiple times, and a crucial requirement is

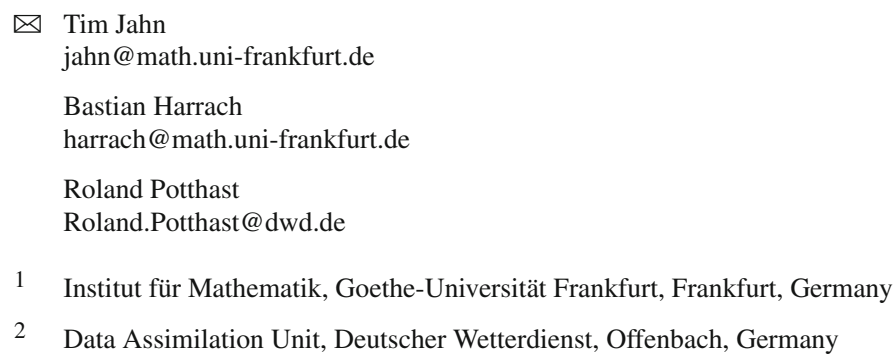


that the solution does not change at least on small time scales. Let us stress that using multiple measurements to decrease the data error is a standard engineering practice under the name 'signal averaging', see, e.g., [27] for an introducing monograph or [20] for a survey article. Examples with low or moderate numbers of measurements (up to a hundred) can be found in [9] or [28] on image averaging or [13] on satellite radar measurements. For the recent first image of a black hole, even up to $10^{9}$ samples were averaged, cf. [1].

The given multiple measurements naturally lead to an estimator of $\hat{y}$, namely the sample mean

$$
\bar{Y}_{n}:=\frac{\sum_{i \leq n} Y_{i}}{n}
$$

But, in general $K^{+} \bar{Y}_{n} \nrightarrow \rightarrow K^{+} \hat{y}$ for $n \rightarrow \infty$, because the generalised inverse (Definition 2.2 of [12]) of $K$ is not continuous. So the inverse is replaced with a family of continuous approximations $\left(R_{\alpha}\right)_{\alpha>0}$, called regularisation, e.g. the Tikhonov regularisation $R_{\alpha}:=\left(K^{*} K+\alpha I d\right)^{-1} K^{*}$, where $I d: \mathscr{X} \rightarrow \mathscr{X}$ is the identity. The regularisation parameter $\alpha$ has to be chosen accordingly to the data $\bar{Y}_{n}$ and the true data error

$$
\delta_{n}^{\text {true }}:=\left\|\bar{Y}_{n}-\hat{y}\right\|,
$$

which is also a random variable. Since $\hat{y}$ is unknown, $\delta_{n}^{\text {true }}$ is also unkown and has to be guessed. Natural guesses are

$$
\delta_{n}^{e s t}:=\frac{1}{\sqrt{n}} \text { or } \delta_{n}^{e s t}:=\frac{\sqrt{\sum_{i \leq n}\left\|Y_{i}-\bar{Y}_{n}\right\|^{2} /(n-1)}}{\sqrt{n}} .
$$

One first natural approach is now to use a (deterministic) regularisation method together with $\bar{Y}_{n}$ and $\delta_{n}^{e s t}$. We are in particular interested in the discrepancy principle [30], wich is known to provide optimal convergence rates (for some $\hat{y}$ ) in the classical deterministic setting. The following main result states, that in a certain sense, the natural approach converges and yields the optimal deterministic rates asymptotically.

Corollary 1 (to Theorems 3 and 4) Assume that $K: \mathscr{X} \rightarrow \mathscr{Y}$ is a compact operator with dense range between Hilbert spaces and that $Y_{1}, Y_{2}, \ldots$ are i.i.d. $\mathscr{Y}-$ valued random variables which fullfill $\mathbb{E}\left[Y_{1}\right]=\hat{y} \in \mathscr{R}(K)$ and $0<\mathbb{E}\left\|Y_{1}-\hat{y}\right\|^{2}<\infty$. Define the Tikhonov regularisation $R_{\alpha}:=\left(K^{*} K+\alpha I d\right)^{-1} K^{*}$ (or the truncated singular value regularisation, or Landweber iteration). Determine $\left(\alpha_{n}\right)_{n}$ through the discrepancy principle using $\delta_{n}^{\text {est }}$ (see Algorithm 1 ). Then $R_{\alpha_{n}} \bar{Y}_{n}$ converges to $K^{+} \hat{y}$ in probability, that is

$$
\mathbb{P}\left(\left\|R_{\alpha_{n}} \bar{Y}_{n}-K^{+} \hat{y}\right\| \leq \varepsilon\right) \rightarrow 1, \quad n \rightarrow \infty, \quad \forall \varepsilon>0
$$

Moreover, if $K^{+} \hat{y}=\left(K^{*} K\right)^{v / 2} w$ with $w \in \mathscr{X}$ and $\|w\| \leq \rho$ for $\rho>0$ and $0<v<v_{0}-1$ (where $v_{0}$ is the qualification of the chosen method, see Assumptions 1 ), then for all $\varepsilon>0$, 


$$
\mathbb{P}\left(\left\|R_{\alpha_{n}} \bar{Y}_{n}-K^{+} \hat{y}\right\| \leq \rho^{\frac{1}{v+1}}\left(\frac{1}{\sqrt{n}}\right)^{\frac{v}{v+1}-\varepsilon}\right) \rightarrow 1, \quad n \rightarrow \infty
$$

Moreover it is shown, that the approach in general does not yield $L^{2}$ convergence $^{1}$ for a naive use of the discrepancy principle, but it does for a priori regularisation. We also discuss quickly, how one has to estimate the error to obtain almost sure convergence.

To solve an inverse problem, as already mentioned, typically some a priori information about the noise is required. This may be, in the classical deterministic case, the knowledge of an upper bound of the noise level, or, in the stochastic case, some knowledge of the error distribution or the restriction to certain classes of distributions, for example to Gaussian distributions. Here we present the first rigorous convergence theory for noisy measurements without any knowledge of the error distribution. The approach can be easily used by everyone, who can measure multiple times.

Stochastic or statistical inverse problems are an active field of research with close ties to high dimensional statistics $[16,17,31]$. In general, there are two approaches to tackle an ill-posed problem with stochastic noise. The Bayesian setting considers the solution of the problem itself as a random quantity, on which one has some a priori knowledge (see [23]). This opposes the frequentist setting, where the inverse problem is assumed to have a deterministic, exact solution $[6,10]$. We are working in the frequentist setting, but we stay close to the classic deterministic theory of linear inverse problems $[12,32,33]$. For statistical inverse problems, typical methods to determine the regularisation parameter are cross validation [34], Lepski's balancing principle [29] or penalised empirical risk minimisation [11]. Modifications of the discrepancy principle were studied recently $[7,8,25,26]$. In [8], it was first shown how to obtain optimal convergence in $L^{2}$ under Gaussian white noise with a modified version of the discrepancy principle.

Another approach is to transfer results from the classical deterministic theory using the Ky-Fan metric, which metrises convergence in probability. In [15,21] it is shown, how to obtain convergence if one knows the Ky-Fan distance between the measurements and the true data. Aspects of the Bakushinskii veto [3] for stochastic inverse problems are discussed in $[4,5,35]$ under assumptions for the noise distribution. In particular, [5] gives an explicit non trivial example for a convergent regularisation, without knowing the exact error level, under Gaussian white noise. We extent this to arbitrary distributions here, if one has multiple measurements.

In the articles mentioned above, the error is usually modelled as a Hilbert space process (such as white noise), thus it is impossible to determine the regularisation parameter directly through the discrepancy principle. This is in contrast to our, more classic error model, where the measurement is an element of the Hilbert space itself. Under the popular assumption that the operator $K$ is Hilbert-Schmidt, one could in principle extend our results to a general Hilbert space process error model (considering the symmetrised equation $K^{*} K \hat{x}=K^{*} \hat{y}$ instead of $K \hat{x}=\hat{y}$, as it is done for example in [8]). But we will postpone the discussion of the white noise case to a follow up paper.

\footnotetext{
1 also called convergence of the integrated mean squared error or root mean squared error
} 
To summarise the connection to the Bakushinskii veto let us state the following. The Bakushinskii veto states that the inverse problem can only be solved with a deterministic regularisation, if the noise level of the data is known. In this article we show, that if one has access to multiple i.i.d. measurements of an unkown distribution, one may use as data the average together with the estimated noise level and one obtains the optimal deterministic rate with high probability, as the number of measurements tends to infinity. That is one can estimate the error from the data. Finally, the measurements potentially contain more information, which is not used here. For example one could estimate the whole covariance structure of one measurement and use this to rescale the measurements and the operator, eventually increasing the relative smoothness of the data. Also one could directly regularise the non-averaged measurements.

In the following section we apply our approach to a priori regularisations and in the main part we consider the widely used discrepancy principle, which is known to work optimal in the classic deterministic theory. After that we quickly show how to choose $\delta_{n}^{e s t}$ to obtain almost sure convergence and we compare the methods numerically.

\section{A priori regularisation}

We use the usual definition that $R_{\alpha}: \mathscr{Y} \rightarrow \mathscr{X}$ is called a linear regularisation, if $R_{\alpha}$ is a bounded linear operator for all $\alpha>0$ and if $R_{\alpha} y \rightarrow K^{+} y$ for $\alpha \rightarrow 0$ for all $y \in \mathscr{D}\left(K^{+}\right)$. A regularisation method is a combination of a regularisation and a parameter choice strategy $\alpha: \mathbb{R}^{+} \times \mathscr{Y} \rightarrow \mathbb{R}^{+}$, such that $R_{\alpha\left(\delta, y^{\delta}\right)} y^{\delta} \rightarrow K^{+} y$ for $\delta \rightarrow 0$, for all $y \in \mathscr{D}\left(K^{+}\right)$and for all $\left(y^{\delta}\right)_{\delta>0} \subset \mathscr{Y}$ with $\left\|y^{\delta}-y\right\| \leq \delta$. The method is called a priori, if the parameter choice does not depend on the data, that is if $\alpha(\delta, y)=\alpha(\delta)$.

The measurements can be formally modelled as realisations of an independent and identically distributed sequence $Y_{1}, Y_{2}, \ldots: \Omega \rightarrow \mathscr{Y}$ of random variables with values in $\mathscr{Y}$, such that $\mathbb{E} Y_{1}=\hat{y} \in \mathscr{D}\left(K^{+}\right)$. Moreover, we require that $0<\mathbb{E}\left\|Y_{1}\right\|^{2}<\infty$, that is the measurements are (almost surely) in the Hilbert space.

In the following we apply the above approach to a priori parameter choice strategies $\alpha\left(y^{\delta}, \delta\right)=\alpha(\delta)$. We restrict to $\delta_{n}^{e s t}=1 / \sqrt{n}$ here, that is we do not estimate the variance here (otherwise the parameter choice would depend on the data). Since then $\delta_{n}^{e s t}$ and hence $\alpha\left(\delta_{n}^{e s t}\right)$ are deterministic, the situation is very easy here and the results are not surprising (see Remark 2).

Theorem 1 (Convergence of a priori regularisation) Assume that $K: \mathscr{X} \rightarrow \mathscr{Y}$ is a bounded linear operator with non-closed range between Hilbert spaces and that $Y_{1}, Y_{2}, \ldots$ are i.i.d. $\mathscr{Y}$-valued random variables which fullfill $\mathbb{E}\left[Y_{1}\right]=\hat{y} \in$ $\mathscr{D}\left(K^{+}\right)$and $0<\mathbb{E}\left\|Y_{1}\right\|^{2}<\infty$. Take an a priori regularisation scheme, with $\alpha(\delta) \stackrel{\delta \rightarrow 0}{\longrightarrow} 0$ and $\left\|R_{\alpha(\delta)}\right\| \delta \stackrel{\delta \rightarrow 0}{\longrightarrow} 0$. Set $\bar{Y}_{n}:=\sum_{i \leq n} Y_{i} / n$ and $\delta_{n}^{e s t}:=n^{-1 / 2}$. Then $\lim _{n \rightarrow \infty} \mathbb{E}\left\|R_{\alpha\left(\delta_{n}^{e s t}\right)} \bar{Y}_{n}-K^{+} \hat{y}\right\|^{2}=0$. 
Proof Because of linearity, $\mathbb{E}\left[R_{\alpha} Y_{1}\right]=R_{\alpha} \mathbb{E}\left[Y_{1}\right]=R_{\alpha} \hat{y}$ and thus by (3)

$$
\mathbb{E}\left\|R_{\alpha} \bar{Y}_{n}-R_{\alpha} \hat{y}\right\|^{2}=\frac{1}{n^{2}} \mathbb{E}\left\|\sum_{i=1}^{n} R_{\alpha}\left(Y_{i}-\hat{y}\right)\right\|^{2}=\frac{\mathbb{E}\left\|R_{\alpha} Y_{1}-R_{\alpha} \hat{y}\right\|^{2}}{n},
$$

since $R_{\alpha} Y_{i} \in \mathscr{R}\left(K^{*}\right)$ where the latter is separable. Therefore, by the bias-variancedecomposition,

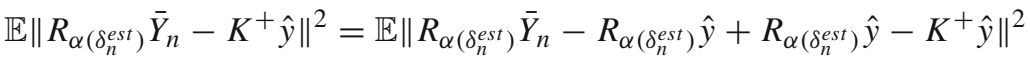

$$
\begin{aligned}
& =\mathbb{E}\left\|R_{\alpha\left(\delta_{n}^{e s t}\right)} \bar{Y}_{n}-R_{\alpha\left(\delta_{n}^{e s t}\right)} \hat{y}\right\|^{2}+\left\|R_{\alpha\left(\delta_{n}^{e s t}\right)} \hat{y}-K^{+} \hat{y}\right\|^{2} \\
& =\frac{\mathbb{E}\left\|R_{\alpha\left(\delta_{n}^{e s t}\right)} Y_{1}-R_{\alpha\left(\delta_{n}^{e s t}\right)} \hat{y}\right\|^{2}}{n}+\left\|R_{\alpha\left(\delta_{n}^{e s t}\right)} \hat{y}-K^{+} \hat{y}\right\|^{2} \\
& \leq \frac{\left\|R_{\alpha\left(\delta_{n}^{e s t}\right)}\right\|^{2}}{n} \mathbb{E}\left\|Y_{1}-\hat{y}\right\|^{2}+\left\|R_{\alpha\left(\delta_{n}^{e s t}\right)} \hat{y}-K^{+} \hat{y}\right\|^{2} \\
& =\left\|R_{\alpha\left(\delta_{n}^{e s t}\right)}\right\|^{2} \delta_{n}^{e s t}{ }^{2} \mathbb{E}\left\|Y_{1}-\hat{y}\right\|^{2}+\left\|R_{\alpha\left(\delta_{n}^{e s t}\right)} \hat{y}-K^{+} \hat{y}\right\|^{2} \\
& \rightarrow 0 \text { for } n \rightarrow \infty \text {. }
\end{aligned}
$$

As in the deterministic case, under additional source conditions we can prove convergence rates. We restrict to regularisations $R_{\alpha}:=F_{\alpha}\left(K^{*} K\right) K^{*}$ defined via the spectral decomposition (see [12]) with the following assumptions for the generating filter.

Assumption $1\left(F_{\alpha}\right)_{\alpha>0}$ is a regularising filter, i.e. a family of piecewise continuous real valued functions on $\left[0,\|K\|^{2}\right]$, continuous from the right, with $\lim _{\alpha \rightarrow 0} F_{\alpha}(\lambda)=\frac{1}{\lambda}$ for all $\lambda \in\left(0,\|K\|^{2}\right]$ and $\lambda F_{\alpha}(\lambda) \leq C_{R}$ for all $\alpha>0$ and all $\lambda \in\left(0,\|K\|^{2}\right]$, where $C_{R}>0$ is some constant. Moreover, it has qualification $\nu_{0}>0$, i.e. $v_{0}$ is maximal such that for all $v \in\left[0, v_{0}\right]$ there exists a constant $C_{v}>0$ with

$$
\sup _{\lambda \in\left(0,\|K\|^{2}\right]} \lambda^{\nu / 2}\left|1-\lambda F_{\alpha}(\lambda)\right| \leq C_{\nu} \alpha^{\nu / 2}
$$

Finally, there is a constant $C_{F}>0$ such that $\left|F_{\alpha}(\lambda)\right| \leq C_{F} / \alpha$ for all $0<\lambda \leq\|K\|^{2}$.

Remark 1 The generating filter of the following regularisation methods fullfill the Assumption 1:

1. Tikhonov regularisation (qualification 2)

2. $n$-times iterated Tikhonov regularisation (qualification $2 n$ ),

3. truncated singular value regularisation (infinite qualification),

4. Landweber iteration (infinite qualification).

Theorem 2 (Rate of convergence of aprioi regularisation) Assume that $K: \mathscr{X} \rightarrow \mathscr{Y}$ is a bounded linear operator with non-closed range between Hilbert spaces and that 
$Y_{1}, Y_{2}, \ldots$ are i.i.d. $\mathscr{Y}$-valued random variables which fullfill $\mathbb{E}\left[Y_{1}\right]=\hat{y} \in \mathscr{D}\left(K^{+}\right)$ and $0<\mathbb{E}\left\|Y_{1}\right\|^{2}<\infty$. Let $R_{\alpha}$ be induced by a filter fullfilling Assumption 1. Set $\bar{Y}_{n}:=\sum_{i \leq n} Y_{i} / n$ and $\delta_{n}^{\text {est }}=n^{-1 / 2}$. Assume that for $0<v \leq v_{0}$ and $\rho>0$ we have that $K^{+} \hat{y}=\left(K^{*} K\right)^{v / 2} w$ for some $w \in \mathscr{X}$ with $\|w\| \leq \rho$. Then iffor constants $0<c<C$,

$$
c\left(\frac{\delta_{n}^{e s t}}{\rho}\right)^{\frac{2}{v+1}} \leq \alpha\left(\delta_{n}^{e s t}\right) \leq C\left(\frac{\delta_{n}^{e s t}}{\rho}\right)^{\frac{2}{v+1}}
$$

we have that $\sqrt{\mathbb{E}\left\|R_{\alpha\left(\delta_{n}^{e s t}\right)} \bar{Y}_{n}-K^{+} \hat{y}\right\|^{2}} \leq C^{\prime} \delta_{n}^{\text {est } \frac{v}{v+1}} \rho^{\frac{1}{v+1}}=\mathscr{O}\left(n^{-\frac{v}{2(v+1)}}\right)$ for some constant $C^{\prime}>0$.

Proof We proceed similiary to the proof of Theorem 1, using additionally Proposition 1 of Sect. 4.

$$
\begin{aligned}
& \mathbb{E}\left\|R_{\alpha\left(\delta_{n}^{e s t}\right)} \bar{Y}_{n}-K^{+} \hat{y}\right\|^{2}=\mathbb{E}\left\|R_{\alpha\left(\delta_{n}^{e s t}\right)} \bar{Y}_{n}-R_{\alpha\left(\delta_{n}^{e s t}\right)} \hat{y}\right\|^{2}+\left\|R_{\alpha\left(\delta_{n}^{e s t}\right)} \hat{y}-K^{+} \hat{y}\right\|^{2} \\
& \leq\left\|R_{\alpha\left(\delta_{n}^{e s t}\right)}\right\|^{2} \delta_{n}^{e s t}{ }^{2} \mathbb{E}\left\|Y_{1}-\hat{y}\right\|^{2}+\left\|R_{\alpha\left(\delta_{n}^{e s t}\right)} \hat{y}-K^{+} \hat{y}\right\|^{2} \\
& \leq C_{R} C_{F} \mathbb{E}\left\|Y_{1}-\hat{y}\right\|^{2} \frac{\delta_{n}^{e s t^{2}}}{\alpha\left(\delta_{n}^{e s t}\right)}+C_{v}^{2} \rho^{2} \alpha\left(\delta_{n}^{e s t}\right)^{v} \\
& \leq \frac{C_{R} C_{F} \mathbb{E}\left\|Y_{1}-\hat{y}\right\|^{2}}{c} \delta_{n}^{\text {est } \frac{-2}{v+1}} \rho^{\frac{2}{v+1}} \delta_{n}^{\text {est } 2} \\
& +C_{v}^{2} C^{v} \delta_{n}^{e s t} \frac{2 v}{v+1} \rho^{\frac{-2 v}{v+1}} \rho^{2} \\
& \leq C^{\prime 2} \delta_{n}^{e s t} \frac{2 v}{v+1} \rho^{\frac{2}{v+1}} \text {. }
\end{aligned}
$$

Remark 2 For separable Hilbert spaces one could alternatively argue as follows: The spaces $\mathscr{X}^{\prime}:=L^{2}(\Omega, \mathscr{X})=\left\{X: \Omega \rightarrow \mathscr{X}: \mathbb{E}\|X\|^{2}<\infty\right\}$ and $\mathscr{Y}^{\prime}:=L^{2}(\Omega, \mathscr{Y})$ are also Hilbert spaces, with scalar products $(X, \tilde{X}) \mathscr{X}^{\prime}:=\sqrt{\mathbb{E}(X, \tilde{X}) \mathscr{X}}$ and $(\cdot, \cdot) \mathscr{Y}^{\prime}$ defined similary. Then $K: \mathscr{X} \rightarrow \mathscr{Y}$ induces naturally a bounded linear operator $K^{\prime}: \mathscr{X}^{\prime} \rightarrow \mathscr{Y}^{\prime}, X \mapsto K X$. Clearly we have that $\hat{y} \in \mathscr{Y}^{\prime}$, and $\left(\bar{Y}_{n}\right)_{n}$ is a sequence in $\mathscr{Y}^{\prime}$ which fullfills

$$
\left\|\bar{Y}_{n}-\hat{y}\right\| \mathscr{Y}^{\prime}:=\sqrt{\left(\bar{Y}_{n}-\hat{y}, \bar{Y}_{n}-\hat{y}\right) \mathscr{Y}^{\prime}}=\sqrt{\frac{\mathbb{E}\left\|Y_{1}-\hat{y}\right\|^{2}}{n}}=\sqrt{\mathbb{E}\left\|Y_{1}-\hat{y}\right\|^{2}} \delta_{n}^{e s t}
$$

and we can use the classic deterministic results for $K^{\prime}: \mathscr{X}^{\prime} \rightarrow \mathscr{Y}^{\prime}$ and $\bar{Y}_{n}$ and $\delta_{n}^{e s t}$.

\section{The discrepancy principle}

In this section we restrict to compact operators with dense range. Note that then $\mathscr{Y}=\overline{\mathscr{R}(K)}$ will be automatically separable. In practice the above parameter choice 
strategies are of limited interest, since they require the knowledge of the abstract smoothness parameters $v$ and $\rho$. The classical discrepancy principle would be to choose $\alpha_{n}$ such that

$$
\left\|\left(K R_{\alpha_{n}}-I d\right) \bar{Y}_{n}\right\| \approx \delta_{n}^{\text {true }}=\left\|\bar{Y}_{n}-\hat{y}\right\|,
$$

which is not possible, because of the unknown $\delta_{n}^{\text {true }}$. So we replace it with our estimator $\delta_{n}^{e s t}$ and implement the discrepancy principle via Algorithm 1 with or without the optional emergency stop.

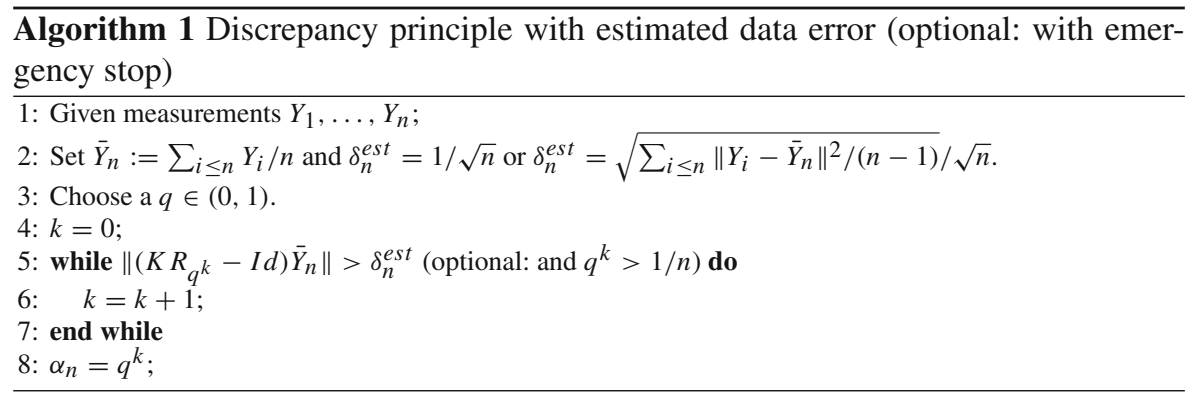

Remark 3 To our knowledge, the idea of an emergency stop first appeared in [8]. It provides a deterministic lower bound for the regularisation parameter, which may avoid overfitting. We use an elementary form of an emergency stop here, which does not require the knowledge of the singular value decomposition of $K$. It would be interesting to see, how more sophisticated versions of the emergency stop worked here, which is not clear to us since in our general setting we cannot rely on the concentration properties of Gaussian noise.

Algorithm 1 will terminate, if we use the emergency stop. Otherwise, we can guarantee that Algorithm 1 terminates, if $K$ has dense image (or equivalently, if $K^{*}$ is injective) and if $\delta_{n}^{e s t}>0$. This is because then $\lim _{\alpha \rightarrow 0} K R_{\alpha}=P_{\overline{\mathscr{R}(K)}}=I d$ pointwise, so $\left\|\left(K R_{q^{k}}-I d\right) \bar{Y}_{n}\right\|<\delta_{n}^{e s t}$ for $k$ large enough. If we decided to use the sample variance, it may happen that $\delta_{n}^{e s t}=0$. But assuming $\mathbb{E}\left\|Y_{1}-\hat{y}\right\|^{2}>0$, it follows that $\mathbb{P}\left(\delta_{n}^{e s t}=0\right)=\mathbb{P}\left(Y_{1}=\cdots=Y_{n}\right) \rightarrow 0$ for $n \rightarrow \infty$ (with exponential rate). If the distribution of $Y_{1}$ posseses a density (with respect to the Gaussian measure for example), then actually $\mathbb{P}\left(Y_{1}=\cdots=Y_{n}\right)=0$ for all $n \in \mathbb{N}$.

Unlike in the previous section, here the $L^{2}$ error will not converge in general, even if $Y_{1}$ has a density. The regularisation parameter $\alpha_{n}$ is now random, since it depends on the potentially bad random data. With a diminishing probability $p$ we are underestimating the data error significantly, and thus the discrepancy principle gives a too small $\alpha$ and we still have $p\left\|R_{\alpha}\right\| \gg 1$ in such a case.

In the following we will need the singular value decomposition of the compact operator $K$ with dense range (see [10]): there exists a monotone sequence $\|K\|=\sigma_{1} \geq$ $\sigma_{2} \geq \cdots>0$ with $\sigma_{l} \rightarrow 0$ for $l \rightarrow \infty$. Moreover there are families of orthonormal 
vectors $\left(u_{l}\right)_{l \in \mathbb{N}}$ and $\left(v_{l}\right)_{l \in \mathbb{N}}$ with $\operatorname{span}\left(u_{l}: l \in \mathbb{N}\right)=\mathscr{Y}, \operatorname{span}\left(v_{l}: l \in \mathbb{N}\right)=\mathscr{N}(K)^{\perp}$ such that $K v_{l}=\sigma_{l} v_{l}$ and $K^{*} u_{l}=\sigma_{l} v_{l}$.

\subsection{A counter example for convergence}

We now show that a naive use of the discrepancy principle, as implemented in Algorithm 1 without emergency stop, may fail to converge in $L^{2}$. To simplify calculations we pick Gaussian noise and the truncated singular value regularisation and we set $\delta_{n}^{e s t}=1 / \sqrt{n}$. We choose $\mathscr{X}:=l^{2}(\mathbb{N})$ with the standard basis $\left\{u_{k}:=(0, \ldots, 0,1,0, \ldots)\right\}$ and consider the diagonal operator

$$
K: l^{2}(\mathbb{N}) \rightarrow l^{2}(\mathbb{N}), \quad u_{l} \mapsto\left(\frac{1}{100}\right)^{\frac{l}{2}} u_{l}
$$

with $\hat{x}=0=\hat{y}=K \hat{x}$. Hence the $\sigma_{l}=(1 / 100)^{\frac{l}{2}}$ are the eigenvalues of $K$ and

$$
R_{\alpha}: l^{2}(\mathbb{N}) \rightarrow l^{2}(\mathbb{N}), \quad y \mapsto \sum_{l: \sigma_{l}^{2} \geq \alpha} \sigma_{l}^{-1}\left(y, u_{l}\right) u_{l}
$$

We assume that the noise is distributed along $y:=\sum_{l \geq 2} 1 / \sqrt{l(l-1)} u_{l}$, so we have that $\sum_{l>n}\left(y, u_{l}\right)^{2}=1 / n$ and thus $y \in l^{2}(\mathbb{N})$. That is we set $\bar{Y}_{n}:=\sum_{i \leq n} Y_{i}=$ $\sum_{i \leq n} Z_{i} y$, where $Z_{i}$ are i.i.d. standard Gaussians. We define $\Omega_{n}:=\left\{Z_{i} \geq 1, i=\right.$ $1 \ldots n\}$, a (very unlikely) event on which we significantly underestimate the true data error. We get that $\mathbb{P}\left(\Omega_{n}\right):=\mathbb{P}\left(Z_{1} \geq 1\right)^{n} \geq 1 / 10^{n}$. Moreover, by the definition of the discrepancy principle

$$
\begin{aligned}
\frac{1}{n} \chi_{\Omega_{n}}=\delta_{n}^{e s t} \chi_{\Omega_{n}} & \geq\left\|\left(K R_{\alpha_{n}}-I d\right) \bar{Y}_{n}\right\|^{2} \chi_{\Omega_{n}}=\left|\bar{Z}_{n}\right|^{2}\left\|\left(K R_{\alpha_{n}}-I d\right) y\right\|^{2} \chi_{\Omega_{n}} \\
& \geq\left\|\left(K R_{\alpha_{n}}-I d\right) y\right\|^{2} \chi_{\Omega_{n}} \\
& =\sum_{l: \sigma_{l}^{2}<\alpha_{n}}\left(y, u_{l}\right)^{2} \chi_{\Omega_{n}}=\sum_{l:(1 / 100)^{i}<\alpha_{n}}\left(y, u_{l}\right)^{2} \chi_{\Omega_{n}} \\
& =\sum_{l>\frac{\log \left(\alpha_{n}\right)}{\log (1 / 100)}}\left(y, u_{l}\right)^{2} \chi_{\Omega_{n}} \geq \frac{\log (1 / 100)}{\log \left(\alpha_{n}\right)} \chi_{\Omega_{n}} \\
\Longrightarrow \alpha_{n} \chi \Omega_{n} & <\frac{1}{100^{n}}
\end{aligned}
$$

It follows that

$$
\begin{aligned}
\mathbb{E}\left\|R_{\alpha_{n}} \bar{Y}_{n}-K^{+} \hat{y}\right\|^{2} & =\mathbb{E}\left\|R_{\alpha_{n}} \bar{Y}_{n}\right\|^{2} \geq \mathbb{E}\left\|R_{\alpha_{n}} \bar{Y}_{n} \chi \Omega_{n}\right\|^{2} \\
& =\mathbb{E}\left[\bar{Z}_{n}^{2}\left\|R_{\alpha_{n}} y \chi \Omega_{n}\right\|\right]^{2} \geq \mathbb{E} \| R_{1 / 100^{n} y \chi \Omega_{n} \|^{2}}
\end{aligned}
$$




$$
\begin{aligned}
& \geq \sum_{l: \sigma_{i}^{2} \geq 1 / 100^{n}} \sigma_{l}^{-2}\left(y, u_{l}\right)^{2} \mathbb{P}\left(\Omega_{n}\right) \geq \frac{1}{10^{n}} \sum_{l \leq n} \sigma_{l}^{-2}\left(y, u_{l}\right)^{2} \\
& \geq \frac{1}{10^{n}} 100^{n}\left(y, u_{n}\right)^{2}=\frac{10^{n}}{n(n-1)} \rightarrow \infty .
\end{aligned}
$$

That is the probability of the events $\Omega_{n}$ is not small enough to compensate the huge error we have on these events, so in the end $\mathbb{E}\left\|R_{\alpha_{n}} \bar{Y}_{n}-K^{+} \hat{y}\right\|^{2} \rightarrow \infty$ for $n \rightarrow \infty$.

\subsection{Convergence in probability of the discrepancy principle}

In this section we show, that the discrepancy principle yields convergence in probability, matching asymptotically the optimal deterministic rate. The proofs of the Theorems 3 and 4 and of Corollary 3 are given in the following section.

Theorem 3 (Convergence of the discrepancy principle) Assume that $K$ is a compact operator with dense range between Hilbert spaces $\mathscr{X}$ and $\mathscr{Y}$ and that $Y_{1}, Y_{2}, \ldots$ are i.i.d. $\mathscr{Y}$-valued random variables with $\mathbb{E} Y_{1}=\hat{y} \in \mathscr{R}(K)$ and $0<\mathbb{E}\left\|Y_{1}-\hat{y}\right\|^{2}<\infty$. Let $R_{\alpha}$ be induced by a filter fullfilling Assumption 1 with $\nu_{0}>1$. Applying Algorithm 1 with or without the emergency stop yields a sequence $\left(\alpha_{n}\right)_{n}$. Then we have that for all $\varepsilon>0$

$$
\mathbb{P}\left(\left\|R_{\alpha_{n}} \bar{Y}_{n}-K^{+} \hat{y}\right\| \leq \varepsilon\right) \stackrel{n \rightarrow \infty}{\longrightarrow} 1,
$$

i.e. $R_{\alpha_{n}} \bar{Y}_{n} \stackrel{\mathbb{P}}{\longrightarrow} K^{+} \hat{y}$.

Remark 4 If one tried to argue as in Remark 1 to show $L^{2}$ convergence one would have to determine the regularisation parameter not as given by Eq. (1), but such that $\mathbb{E}\left\|\left(K R_{\alpha}-I d\right) \bar{Y}_{n}\right\|^{2} \approx \delta_{n}^{e s t}$, which is not practicable since we cannot calculate the expectation on the left hand side.

The popularity of the discrepancy principles is a result of the fact that it guarantees optimal convergence rates under an additional source condition: Assuming that there is a $0<v \leq v_{0}-1$ (where $v_{0}$ is the qualification of the chosen regularisation method) such that $K^{+} \hat{y}=\left(K^{*} K\right)^{\frac{v}{2}} w$ for a $w \in \mathscr{X}$ with $\|w\| \leq \rho$, then

$$
\sup _{y^{\delta}:\left\|y^{\delta}-\hat{y}\right\| \leq \delta}\left\|R_{\alpha\left(y^{\delta}, \delta\right)} y^{\delta}-K^{+} \hat{y}\right\| \leq C \rho^{\frac{1}{v+1}} \delta^{\frac{v}{v+1}}
$$

for some constant $C>0$. The next theorem shows a concentration result for the discrepancy principle as implemented in Algorithm 1, with a bound similiar to (2).

Theorem 4 (Rate of convergence of the discrepancy principle) Assume that $K$ is a compact operator with dense range between Hilbert spaces $\mathscr{X}$ and $\mathscr{Y}$. Moreover, $Y_{1}, Y_{2}, \ldots$ are i.i.d. $\mathscr{Y}$-valued random variables with $\mathbb{E} Y_{1}=\hat{y} \in \mathscr{R}(K)$ and $0<$ $\mathbb{E}\left\|Y_{1}-\hat{y}\right\|^{2}<\infty$. Let $R_{\alpha}$ be induced by a filter fullfilling Assumption 1 with $v_{0}>1$. Moreover, assume that there is a $0<v \leq v_{0}-1$ and a $\rho>0$ such that $K^{+} \hat{y}=$ 
$\left(K^{*} K\right)^{v / 2} w$ for some $w \in \mathscr{X}$ with $\|w\| \leq \rho$. Applying Algorithm 1 with or without the emergency stop yields a sequence $\left(\alpha_{n}\right)_{n \in \mathbb{N}}$. Then there is a constant $L$, such that

$$
\mathbb{P}\left(\left\|R_{\alpha_{n}} \bar{Y}_{n}-K^{+} \hat{y}\right\| \leq L \rho^{\frac{1}{v+1}} \max \left\{\delta_{n}^{\text {est } \frac{v}{v+1}}, \delta_{n}^{\text {true } \frac{v}{v+1}}\left(\delta_{n}^{\text {true }} / \delta_{n}^{e s t}\right)^{\frac{1}{v+1}}\right\}\right) \stackrel{n \rightarrow \infty}{\longrightarrow} 1 .
$$

We deduce a deterministic bound for $\left\|R_{\alpha_{n}} \bar{Y}_{n}-K^{+} \hat{y}\right\|$ (for $n$ large).

Corollary 2 Under the assumptions of Theorem 4, for all $\varepsilon>0$ it holds that

$$
\mathbb{P}\left(\left\|R_{\alpha_{n}} \bar{Y}_{n}-K^{+} \hat{y}\right\| \leq \rho^{\frac{1}{v+1}}\left(\frac{1}{\sqrt{n}}\right)^{\frac{v}{v+1}-\varepsilon}\right) \stackrel{n \rightarrow \infty}{\longrightarrow} 1
$$

Proof (Corollary 2) By the second assertion in Lemma 1 and Markov's inequality, for any $c, \varepsilon>0$,

$$
\lim _{n \rightarrow \infty} \mathbb{P}\left(\delta_{n}^{\text {est }}, \delta_{n}^{\text {true }} \leq c n^{-\frac{1}{2}+\varepsilon}\right)=1
$$

The ad hoc emergency stop $\alpha_{n}>1 / n$, additionally assures, that the $L^{2}$ error will not explode (unlike in the counter example of the previous subsection). Under the assumption that $\mathbb{E}\left\|Y_{1}-\hat{y}\right\|^{4}<\infty$, one can guarantee, that the $L^{2}$ error will converge.

Corollary 3 Under the assumptions of Theorem 3, consider the sequence $\alpha_{n}$ determined by Algorithm 1 with emergency stop. Then there is a constant $C$ such that $\mathbb{E}\left\|R_{\alpha_{n}} \bar{Y}_{n}-K^{+} \hat{y}\right\|^{2} \leq C$ for all $n \in \mathbb{N}$. If additionally $\mathbb{E}\left\|Y_{1}-\hat{y}\right\|^{4}<\infty$, then it holds that $\mathbb{E}\left\|R_{\alpha_{n}} \bar{Y}_{n}-K^{+} \hat{y}\right\|^{2} \rightarrow 0$ for $n \rightarrow \infty$.

\subsection{Almost sure convergence}

The results so far delievered either convergence in probability or convergence in $L^{2}$. We give a short remark how one can obtain almost sure convergence. Roughly speaking, one has to multiply a $\sqrt{\log \log n}$ term to $\delta_{n}^{e s t}$. This is a simple consequence of the following theorem

Theorem 5 (Law of the iterated logarithm) Assume that $Y_{1}, Y_{2}, \ldots$ is an i.i.d sequence with values in some seperable Hilbert space $\mathscr{Y}$. Moreover, assume that $\mathbb{E} Y_{1}=0$ and $\mathbb{E}\left\|Y_{1}\right\|^{2}<\infty$. Then we have that

$$
\mathbb{P}\left(\limsup _{n \rightarrow \infty} \frac{\left\|\sum_{i \leq n} Y_{i}\right\|}{\sqrt{2 \mathbb{E}\left\|Y_{1}\right\|^{2} n \log \log n}} \leq 1\right)=1
$$

Proof This is a simple consequence of Corollary 8.8 in [24]. 
So if $\mathbb{E} Y_{1}=\hat{y} \in \mathscr{Y}$ we have for $\delta_{n}^{\text {true }}=\left\|\bar{Y}_{n}-\hat{y}\right\|$

$$
\mathbb{P}\left(\limsup _{n \rightarrow \infty} \frac{\sqrt{n} \delta_{n}^{\text {true }}}{\sqrt{2 \mathbb{E}\left\|Y_{1}-\hat{y}\right\|^{2} \log \log n}} \leq 1\right)=1 \text {, }
$$

that is, with probability 1 it holds that $\delta_{n}^{\text {true }} \leq \sqrt{\frac{2 \mathbb{E}\left\|Y_{1}-\hat{y}\right\|^{2} \log \log n}{n}}$ for $n$ large enough. Consequently, for some $\tau>1$ the estimator should be

$$
\delta_{n}^{e s t}:=\tau s_{n} \sqrt{\frac{2 \log \log n}{n}},
$$

where $s_{n}$ is the square root of the sample variance. Since $\mathbb{P}\left(\lim _{n \rightarrow \infty} s_{n}^{2}=\mathbb{E} \| Y_{1}-\right.$ $\left.\hat{y} \|^{2}\right)=1$ and $\tau>1$ it holds that $\sqrt{\mathbb{E}\left\|Y_{1}-\hat{y}\right\|} \leq \tau s_{n}$ for $n$ large enough with probability 1 and thus $\delta_{n}^{\text {true }} \leq \delta_{n}^{\text {est }}$ for $n$ large enough with probability 1 . In other words, there is an event $\Omega_{0} \subset \Omega$ with $\mathbb{P}\left(\Omega_{0}\right)=1$ such that for any $\omega \in \Omega_{0}$ there is a $N(\omega) \in \mathbb{N}$ with $\delta_{n}^{\text {true }}(\omega) \leq \delta_{n}^{e s t}(\omega)$ for all $n \geq N(\omega)$. So we can use $\bar{Y}_{n}$ and $\delta_{n}^{\text {est }}$ together with any deterministic regularisation method to get almost sure convergence.

\section{Proofs of Theorem 3 and 4}

\subsection{Proofs without emergency case}

We will multiple times use the Pythagorean theorem for independent separable Hil-bert space valued random variables $Z_{i}$ with $\mathbb{E}\left\|Z_{i}\right\|^{2}<\infty$ and $\mathbb{E} Z_{i}=0$,

$$
\mathbb{E}\left\|\sum_{i=1}^{n} Z_{i}\right\|^{2}=\sum_{i=1}^{n} \sum_{l, l^{\prime}=1}^{\infty} \mathbb{E}\left[\left(Z_{i}, e_{l}\right)\left(Z_{i}, e_{l^{\prime}}\right)\right]=\sum_{i=1}^{n} \mathbb{E}\left[\sum_{j=1}^{\infty}\left(Z_{i}, e_{j}\right)^{2}\right]=\sum_{i=1}^{n} \mathbb{E}\left\|Z_{i}\right\|^{2},
$$

where $\left(e_{l}\right)_{l \in \mathbb{N}}$ is an orthonormal basis. Based on this, the central ingridient will be the following lemma, which strengthens the pointwise worst case error bound $\|\left(K R_{\alpha}-\right.$ $\operatorname{Id})\left(\bar{Y}_{n}-\hat{y}\right) \| \leq C_{0} \delta_{n}^{\text {true }}$ in some sense.

Lemma 1 For all $\varepsilon>0$ and (deterministic) sequences $\left(q_{n}\right)_{n \in \mathbb{N}}$ with $q_{n}>0$ and $\lim _{n \rightarrow \infty} q_{n}=0$, it holds that

$$
\mathbb{P}\left(\left\|\left(K R_{q_{n}}-I d\right)\left(\bar{Y}_{n}-\hat{y}\right)\right\| \geq \varepsilon / \sqrt{n}\right) \rightarrow 0
$$

and

$$
\mathbb{P}\left(\left|\sqrt{n} \delta_{n}^{e s t}-\gamma\right| \geq \varepsilon\right) \rightarrow 0
$$

for $n \rightarrow \infty$, where $\gamma=1$ or $\gamma=\sqrt{\mathbb{E}\left\|Y_{1}-\hat{y}\right\|^{2}}$, depending on if we used the sample variance or not. 
Proof By Tschebyscheff's inequality and (3)

$$
\begin{aligned}
\mathbb{P}\left(\left\|\left(K R_{q_{n}}-I d\right)\left(\bar{Y}_{n}-\hat{y}\right)\right\| \geq \varepsilon / \sqrt{n}\right) & \leq \frac{n}{\varepsilon^{2}} \mathbb{E}\left\|\left(K R_{q_{n}}-I d\right)\left(\bar{Y}_{n}-\hat{y}\right)\right\|^{2} \\
& =\frac{1}{\varepsilon^{2}} \mathbb{E}\left\|\left(K R_{q_{n}}-I d\right)\left(Y_{1}-\hat{y}\right)\right\|^{2}
\end{aligned}
$$

Since $K$ has dense range, $K R_{q_{n}}-I d$ converges to 0 pointwise for $n \rightarrow \infty$ and it follows that $\left(K R_{q_{n}}-I d\right)\left(Y_{1}-\hat{y}\right)$ also converges pointwise to 0 . By inequality (6) of Proposition 1 below, $\left\|\left(K R_{q_{n}}-I d\right)\left(Y_{1}-\hat{y}\right)\right\|^{2} \leq C_{0}\left\|Y_{1}-\hat{y}\right\|^{2}$, so $\mathbb{E} \|\left(K R_{q_{n}}-\right.$ $I d)\left(Y_{1}-\hat{y}\right) \|^{2} \rightarrow 0$ for $n \rightarrow \infty$ by the dominated convergence theorem. The second assertion only needs a proof for $\gamma=\sqrt{\mathbb{E}\left\|Y_{1}-\hat{y}\right\|^{2}}$ and then

$$
\begin{aligned}
n \delta_{n}^{e s t^{2}} & =\frac{1}{n-1} \sum_{i=1}^{n}\left\|Y_{i}-\bar{Y}_{n}\right\|^{2}=\frac{n}{n-1}\left(\frac{1}{n} \sum_{i=1}^{n}\left\|Y_{i}\right\|^{2}-\left\|\bar{Y}_{n}\right\|^{2}\right) \\
& \rightarrow \mathbb{E}\left\|Y_{1}\right\|^{2}-\|\hat{y}\|^{2}=\mathbb{E}\left\|Y_{1}-\hat{y}\right\|^{2}=\gamma^{2}
\end{aligned}
$$

almost surely (thus in particular in probability) for $n \rightarrow \infty$ by the strong law of large numbers (Corollary 7.10 in [24]) and the bias-variance-decomposition. Therefore $\sqrt{n} \delta_{n}^{e s t} \rightarrow \gamma$ in probability for $n \rightarrow \infty$.

For convergence in probability it does not matter how large the error is on sets with diminishing probability and with Lemma 1 we will show, that the probability of certain 'good events' is 1 in the limit of infinitely many measurements.

Define for $q \in(0,1)$ (as chosen in Algorithm 1)

$$
\begin{aligned}
\psi_{q}: \mathbb{R}^{+} & \rightarrow\left\{q^{k}: k \in \mathbb{N}_{0}\right\} \\
\alpha & \mapsto \max \left\{q^{k}: q^{k} \leq \alpha\right\} .
\end{aligned}
$$

So $\min (q \alpha, 1) \leq \psi_{q}(\alpha) \leq \alpha$ and by definition, if $\left\|\left(K R_{\psi_{q}(\alpha)}-I d\right) \bar{Y}_{n}\right\|<\delta_{n}^{e s t}$, it holds that $\alpha_{n} \geq \min (q \alpha, 1)$, where $\alpha_{n}$ is the output of Algorithm 1 .

We will also need some well known properties of regularisations defined by filters which fullfill Assumption 1. These are mostly easy modifications from [12].

Proposition 1 The constants in the following are defined as in Assumption 1. We assume, that $K$ is bounded and linear with non-closed range. Assume that $\left(R_{\alpha}\right)_{\alpha>0}$ is induced by a regularising filter fullfilling $\left|F_{\alpha}(\lambda)\right| \leq C_{F} / \alpha$ for all $0<\lambda \leq\|K\|^{2}$. Then

$$
\begin{aligned}
\left\|R_{\alpha}\right\| & \leq \sqrt{C_{R} C_{F}} / \sqrt{\alpha} \\
\left\|I d-K R_{\alpha}\right\| & \leq C_{0}
\end{aligned}
$$


for all $\alpha>0$, with $C_{0} \geq 1$. If moreover, the filter has qualification $\nu_{0}>0$ and there is a $w \in \mathscr{X}$ with $\|w\| \leq \rho$ such that $K^{+} \hat{y}=\left(K^{*} K\right)^{\frac{v}{2}} w$ for some $0<v \leq v_{0}$, then

$$
\begin{aligned}
& \left\|R_{\alpha} \hat{y}-K^{+} \hat{y}\right\| \leq C_{v} \rho \alpha^{v / 2} \\
& \left\|R_{\alpha} \hat{y}-K^{+} \hat{y}\right\| \leq\left\|K R_{\alpha} \hat{y}-K K^{+} \hat{y}\right\|^{\frac{v}{v+1}} C_{0}^{\frac{1}{v+1}} \rho^{\frac{1}{v+1}}
\end{aligned}
$$

for all $\alpha>0$. If additionally, $v_{0} \geq v+1>1$, then

$$
\left\|K R_{\alpha} \hat{y}-K K^{+} \hat{y}\right\| \leq C_{v+1} \rho \alpha^{\frac{v+1}{2}} .
$$

Moreover, if $K$ is compact, than for all $x \in \mathscr{X}$ there is a function $g: \mathbb{R}^{+} \rightarrow \mathbb{R}^{+}$with $g(\alpha) \rightarrow \infty$ for $\alpha \rightarrow 0$, such that

$$
\lim _{\alpha \rightarrow 0}\left\|\left(K R_{\psi_{q}(\alpha g(\alpha))}-I d\right) K x\right\| / \sqrt{\alpha}=0,
$$

where $\psi_{q}$ is given in (4).

Proof (Proposition 1) (5) and (8) are shown in the proofs of Theorem 4.2 and Theorem 4.17 in [12]. (7) and (8) are Theorem 4.3 in [12]. (6) follows directly from Assumption 1.

For (10), let $x \in \mathscr{X}$ be fixed and set

$$
\tilde{g}(\alpha):=\sup \left\{t>0:\left\|\left(K R_{\psi_{q}(\alpha t)}-I d\right) K x\right\| / \sqrt{\alpha} \leq t^{-1}\right\} .
$$

W.1.o.g. $\tilde{g}$ is finite for any $\alpha>0$. Now we first show that

$$
\lim _{\alpha \rightarrow 0}\left\|\left(K R_{\alpha}-I d\right) K x\right\| / \sqrt{\alpha}=0 .
$$

We mimic the proof of Theorem 3.1.17 of [31] and set $\varepsilon>0$. We fix $L$, such that $C_{1}^{2} \sum_{l=L+1}^{\infty}\left(\hat{x}, v_{l}\right)^{2}<\varepsilon$. Then

$$
\begin{aligned}
\left\|\left(K R_{\alpha}-I d\right) K \hat{x}\right\|^{2} / \alpha & =\sum_{l=1}^{\infty}\left(F_{\alpha}\left(\sigma_{l}^{2}\right) \sigma_{l}^{2}-1\right)^{2} \frac{\sigma_{l}^{2}}{\alpha}\left(\hat{x}, v_{l}\right)^{2} \\
& \leq\left(\sup _{\lambda>0} \lambda^{\frac{\nu_{0}}{2}}\left|F_{\alpha}(\lambda) \lambda-1\right|\right)^{2}\|\hat{x}\|^{2} \sum_{l=1}^{L} \frac{\sigma_{l}^{2\left(1-\nu_{0}\right)}}{\alpha} \\
& +\left(\sup _{\lambda>0} \lambda^{\frac{1}{2}}\left|F_{\alpha}(\lambda) \lambda-1\right|\right)^{2} \frac{\sum_{l=L+1}^{\infty}\left(\hat{x}, v_{l}\right)^{2}}{\alpha} \\
& \leq C_{v_{0}}^{2} L \sigma_{L}^{2\left(1-v_{0}\right)}\|\hat{x}\|^{2} \alpha^{\nu_{0}-1}+C_{1}^{2} \sum_{l=L+1}^{\infty}\left(\hat{x}, v_{l}\right)^{2}<2 \varepsilon
\end{aligned}
$$


for all $\alpha<\left(\varepsilon^{-1} C_{v_{0}}^{2} L \sigma_{L}^{2\left(1-v_{0}\right)}\|\hat{x}\|^{2}\right)^{-\frac{1}{v_{0}-1}}$, therefore $\left\|\left(K R_{\alpha}-I d\right) K x\right\| / \sqrt{\alpha}=0$ for $\alpha \rightarrow 0$. So for any $t>0$

$$
\left\|\left(K R_{\psi_{q}(\alpha t)}-I d\right) K x\right\| / \sqrt{\alpha}=\sqrt{\frac{\psi_{q}(\alpha t)}{\alpha}}\left\|\left(K R_{\psi_{q}(\alpha t)}-I d\right) K x\right\| / \sqrt{\psi_{q}(\alpha t)} \leq \frac{1}{t}
$$

for $\alpha$ small enough, because of (11) and since $\psi_{q}(\alpha t) \leq \alpha t$. So $\tilde{g}(\alpha) \rightarrow \infty$ for $\alpha \rightarrow 0$ and by definition of $\tilde{g}$ the claim holds for $g(\alpha):=\tilde{g}(\alpha)-1$ ( $g$ is well defined for $\alpha$ small enough).

Proof (Theorem 4) Set $q_{n}:=\psi_{q}\left(b_{n}\right)$ where $b_{n}:=\left(\frac{1}{\rho} \frac{\gamma}{4 C_{v+1} \sqrt{n}}\right)^{\frac{2}{v+1}}$ with $\gamma=1$ or $\gamma=\sqrt{\mathbb{E}\left\|Y_{1}-\hat{y}\right\|^{2}}$, depending on if we used the sample variance or not, and $\psi_{q}$ given in (4). Define

$$
\Omega_{n}:=\Omega_{n}\left(q_{n}, \gamma\right):=\left\{\left|\sqrt{n} \delta_{n}^{e s t}-\gamma\right|<\gamma / 2,\left\|\left(K R_{q_{n}}-I d\right)\left(\bar{Y}_{n}-\hat{y}\right)\right\|<\gamma / \sqrt{16 n}\right\} .
$$

Then by (9) and since $q_{n} \leq b_{n}$,

$$
\begin{aligned}
\left\|\left(K R_{q_{n}}-I d\right) \bar{Y}_{n}\right\| \chi_{\Omega_{n}} & \leq\left\|\left(K R_{q_{n}}-I d\right) \hat{y}\right\| \chi_{\Omega_{n}}+\left\|\left(K R_{q_{n}}-I d\right)\left(\bar{Y}_{n}-\hat{y}\right)\right\| \chi_{\Omega_{n}} \\
& \leq C_{\nu+1} \rho b_{n}^{\frac{v+1}{2}} \chi \Omega_{n}+\frac{\gamma}{4 \sqrt{n}} \chi \Omega_{n}=\frac{\gamma}{2 \sqrt{n}} \chi \Omega_{n}<\delta_{n}^{e s t} \chi_{\Omega_{n}}
\end{aligned}
$$

so $\alpha_{n} \chi \Omega_{n} \geq q b_{n} \chi \Omega_{n} \geq q\left(\frac{\delta_{n}^{e s t}}{6 C_{v+1}}\right)^{\frac{2}{v+1}} \chi \Omega_{n}$ for $n$ large enough. By (6), (8) and since $K$ has dense image,

$$
\begin{aligned}
\left\|R_{\alpha_{n}} \hat{y}-K^{+} \hat{y}\right\| & \leq\left\|K R_{\alpha_{n}} \hat{y}-K K^{+} \hat{y}\right\| \frac{v}{v+1} C_{0}^{\frac{1}{v+1}} \rho^{\frac{1}{v+1}}=\left\|K R_{\alpha_{n}} \hat{y}-\hat{y}\right\|^{\frac{v}{v+1}} C_{0}^{\frac{1}{v+1}} \rho^{\frac{1}{v+1}} \\
& \leq\left(\left\|\left(K R_{\alpha_{n}}-I d\right) \bar{Y}_{n}\right\|+\left\|\left(K R_{\alpha_{n}}-I d\right)\left(\hat{y}-\bar{Y}_{n}\right)\right\|\right)^{\frac{v}{v+1}} C_{0}^{\frac{1}{v+1}} \rho^{\frac{1}{v+1}} \\
& \leq\left(\delta_{n}^{e s t}+\left\|\left(K R_{\alpha_{n}}-I d\right)\left(\hat{y}-\bar{Y}_{n}\right)\right\|\right)^{\frac{v}{v+1}} C_{0}^{\frac{1}{v+1}} \rho^{\frac{1}{v+1}} \\
& \leq\left(\delta_{n}^{e s t}+C_{0} \delta_{n}^{\text {true }}\right)^{\frac{v}{v+1}} C_{0}^{\frac{1}{v+1}} \rho^{\frac{1}{v+1}} \leq\left(\delta_{n}^{e s t}+\delta_{n}^{\text {true }}\right)^{\frac{v}{v+1}} C_{0} \rho^{\frac{1}{v+1}}
\end{aligned}
$$

Finally,

$$
\begin{aligned}
& \left\|R_{\alpha_{n}} \bar{Y}_{n}-K^{+} \hat{y}\right\| \chi_{\Omega_{n}} \\
& \quad \leq\left\|R_{\alpha_{n}} \hat{y}-K^{+} \hat{y}\right\| \chi_{\Omega_{n}}+\left\|R_{\alpha_{n}} \bar{Y}_{n}-R_{\alpha_{n}} \hat{y}\right\| \chi_{\Omega_{n}} \\
& \quad \leq\left(\delta_{n}^{e s t}+\delta_{n}^{\text {true }}\right)^{\frac{v}{v+1}} C_{0} \rho^{\frac{1}{v+1}} \chi_{\Omega_{n}}+\sqrt{C_{R} C_{F}} \frac{\delta_{n}^{\text {true }}}{\sqrt{\alpha_{n}}} \chi_{\Omega_{n}}
\end{aligned}
$$




$$
\begin{aligned}
& \leq\left(2 \max \left(\delta_{n}^{\text {est }}, \delta_{n}^{\text {true }}\right)\right)^{\frac{v}{v+1}} C_{0} \rho^{\frac{1}{v+1}} \chi \Omega_{n}+\sqrt{C_{R} C_{F}} \rho^{\frac{1}{v+1}}\left(\frac{6 C_{v+1}}{\delta_{n}^{e s t}}\right)^{\frac{1}{v+1}} \frac{\delta_{n}^{\text {true }}}{\sqrt{q}} \chi \Omega_{n} \\
& \leq L \max \left\{\delta_{n}^{\text {est } \frac{v}{v+1}}, \delta_{n}^{\text {true } \frac{v}{v+1}}\left(\frac{\delta_{n}^{\text {true }}}{\delta_{n}^{\text {est }}}\right)^{\frac{1}{v+1}}\right\},
\end{aligned}
$$

with $L:=2^{\frac{v}{v+1}} C_{0} \rho^{\frac{1}{v+1}}+\sqrt{C_{R} C_{F} / q}\left(6 C_{v+1}\right)^{\frac{1}{v+1}}$ and the proof is finished, because $\mathbb{P}\left(\Omega_{n}\right) \rightarrow 1$ for $n \rightarrow \infty$ by Lemma 1 .

Proof (Proof of Theorem 3) W.l.o.g. we may assume that there are arbitrarily large $l \in$ $\mathbb{N}$ with $\left(\hat{y}, u_{l}\right) \neq 0$, since otherwise we could apply Theorem 4 with any $v>0$. Let $\varepsilon^{\prime}>0$. Then there is a $L \in \mathbb{N}$ such that $\left(\hat{y}, u_{L}\right) \neq 0$ and $\left(F_{q^{k}}\left(\sigma_{L}^{2}\right) \sigma_{L}^{2}-1\right)^{2}>1 / 2$ for all $k \in \mathbb{N}_{0}$ with $q^{k} \geq \varepsilon^{\prime}$ (because the $F_{q^{k}}$ are bounded and $\sigma_{l} \rightarrow 0$ for $l \rightarrow \infty$ ). Set

$$
\Omega_{n}:=\left\{\left|\sqrt{n} \delta_{n}^{e s t}-\gamma\right|<\gamma,\left(\bar{Y}_{n}, u_{L}\right)^{2} \geq\left(\hat{y}, u_{L}\right)^{2} / 2\right\}
$$

Then for $n \geq 16 \gamma^{2} /\left(\hat{y}, u_{L}\right)^{2}$,

$$
\begin{aligned}
\delta_{n}^{e s t} \chi_{\Omega_{n}} & \leq \frac{2 \gamma}{\sqrt{n}} \chi_{\Omega_{n}}<\sqrt{\frac{\left(\hat{y}, u_{L}\right)^{2}}{4}} \chi_{\Omega_{n}} \leq \sqrt{\left(F_{q^{k}}\left(\sigma_{L}^{2}\right) \sigma_{L}^{2}-1\right)^{2}\left(\bar{Y}_{n}, u_{L}\right)^{2}} \chi_{\Omega_{n}} \\
& \leq \sqrt{\sum_{l=1}^{\infty}\left(F_{q^{k}}\left(\sigma_{l}^{2}\right) \sigma_{l}^{2}-1\right)^{2}\left(\bar{Y}_{n}, u_{l}\right)^{2}} \chi_{\Omega_{n}}=\left\|\left(K R_{q^{k}}-I d\right) \bar{Y}_{n}\right\| \chi \Omega_{n}
\end{aligned}
$$

for all $k \in \mathbb{N}_{0}$ with $q^{k} \geq \varepsilon^{\prime}$. Thus for $\Omega_{n}$ given in (14)

$$
\lim _{n \rightarrow \infty} \mathbb{P}\left(\alpha_{n} \leq \varepsilon^{\prime}\right) \geq \lim _{n \rightarrow \infty} \mathbb{P}\left(\Omega_{n}\right)=1
$$

by Lemma 1 and since $\left(\bar{Y}_{n}, u_{L}\right)=\sum_{i=1}^{n}\left(Y_{i}, u_{L}\right) / n \rightarrow \mathbb{E}\left(Y_{1}, u_{L}\right)=\left(\hat{y}, u_{L}\right) \neq 0$ almost surely for $n \rightarrow \infty$. Set $q_{n}:=\psi_{q}\left(b_{n}\right)$ with $b_{n}:=n^{-1} g\left(n^{-1}\right)$ and $g$ and $\psi_{q}$ given in (4) and (10). Define

$$
\Omega_{n}:=\left\{\left|\sqrt{n} \delta_{n}^{e s t}-\gamma\right|<\gamma / 2,\left\|\left(K R_{q_{n}}-I d\right)\left(\bar{Y}_{n}-\hat{y}\right)\right\|<\gamma / 4 \sqrt{n}\right\} .
$$

Then for $n$ large enough (such that $\left\|\left(K R_{q_{n}}-I d\right) \hat{y}\right\| \sqrt{n} \leq \gamma / 4$, see (10) with $\left.\alpha=n^{-1}\right)$,

$$
\begin{aligned}
& \left\|\left(K R_{q_{n}}-I d\right) \bar{Y}_{n}\right\| \chi \Omega_{\Omega_{n}} \\
& \leq \frac{1}{\sqrt{n}} \sqrt{n}\left\|\left(K R_{q_{n}}-I d\right) \hat{y}\right\| \chi \Omega_{n}+\left\|\left(K R_{q_{n}}-I d\right)\left(\bar{Y}_{n}-\hat{y}\right)\right\| \chi \Omega_{n} \\
& \leq \frac{\gamma}{4 \sqrt{n}} \chi \Omega_{n}+\frac{\gamma}{4 \sqrt{n}} \chi_{\Omega_{n}} \leq \frac{\gamma}{2 \sqrt{n}} \chi \Omega_{n} \leq \delta_{n}^{e s t} \chi \Omega_{n} .
\end{aligned}
$$


That is $\alpha_{n} \chi \Omega_{n} \geq q b_{n} \chi \Omega_{n} \geq q n^{-1} g\left(n^{-1}\right) \chi \Omega_{n}$ for $n$ large enough. Finally set

$$
\tilde{\Omega}_{n}:=\left\{\delta_{n}^{\text {true }} \leq \sqrt{\frac{\sqrt{g\left(n^{-1}\right)}}{n}},\left\|R_{\alpha_{n}} \hat{y}-K^{+} \hat{y}\right\| \leq \frac{\varepsilon}{2}\right\} \cap \Omega_{n},
$$

with $\Omega_{n}$ given in (16). So $\mathbb{P}\left(\tilde{\Omega}_{n}\right) \rightarrow 1$ for $n \rightarrow \infty$, since $\mathbb{P}\left(\delta_{n}^{\text {true }} \leq \sqrt{\sqrt{g\left(n^{-1}\right)} / n}\right) \rightarrow$ 1 , because of $g\left(n^{-1}\right) \rightarrow \infty, \mathbb{P}\left(\Omega_{n}\right) \rightarrow 1$ by Lemma 1 and $\mathbb{P}\left(\left\|R_{\alpha_{n}} \hat{y}-K^{+} \hat{y}\right\| \leq \frac{\varepsilon}{2}\right) \rightarrow$ 1 by (15) ( $\varepsilon^{\prime}>0$ is arbitrary). Thus for $n$ large enough (so that $C_{R} C_{F} / q \sqrt{g\left(n^{-1}\right)} \leq$ $\left.\frac{\varepsilon^{2}}{4}\right)$

$$
\begin{aligned}
\left\|R_{\alpha_{n}} \bar{Y}_{n}-K^{+} \hat{y}\right\| \chi_{\tilde{\Omega}_{n}} & \leq\left\|R_{\alpha_{n}} \hat{y}-K^{+} \hat{y}\right\| \chi_{\tilde{\Omega}_{n}}+\left\|R_{\alpha_{n}}\left(\bar{Y}_{n}-\hat{y}\right)\right\| \chi_{\tilde{\Omega}_{n}} \\
& \leq \frac{\varepsilon}{2}+\sqrt{\frac{C_{R} C_{F}}{\alpha_{n}}} \delta_{n}^{\text {true }} \chi_{\tilde{\Omega}_{n}} \leq \frac{\varepsilon}{2}+\sqrt{\frac{C_{R} C_{F}}{q \sqrt{g\left(n^{-1}\right)}}} \leq \varepsilon
\end{aligned}
$$

and $\mathbb{P}\left(\left\|R_{\alpha_{n}} \bar{Y}_{n}-K^{+} \hat{y}\right\| \leq \varepsilon\right) \geq \mathbb{P}\left(\tilde{\Omega}_{n}\right) \rightarrow 1$ for $n \rightarrow \infty$.

\subsection{Proofs for the emergency stop case}

Again, denote by $\alpha_{n}$ the output of Algorithm 1 without the emergency stop. For the emergency stop, we have to consider $\left\|R_{\max \left\{\alpha_{n}, 1 / n\right\}} \bar{Y}_{n}-K^{+} \hat{y}\right\|$. It suffices to show that $\mathbb{P}\left(\alpha_{n} \geq 1 / n\right) \rightarrow 1$ for $n \rightarrow \infty$.

First assume that $K^{+} \hat{y}=\left(K^{*} K\right)^{\frac{v}{2}} w$ for some $w \in \mathscr{X}$ with $\|w\| \leq \rho$ and $0<$ $v \leq v_{0}-1$. With (13) it follows that

$$
\mathbb{P}\left(\alpha_{n} \geq q\left(\frac{\gamma}{4 \rho C_{\nu+1} \sqrt{n}}\right)^{\frac{2}{\nu+1}}\right) \geq \mathbb{P}\left(\Omega_{n}\right) \rightarrow 1
$$

for $n \rightarrow \infty$, with $\Omega_{n}$ given in (12). Otherwise, if there are no such $v, \rho$ and $w$, then (17) implies that for all $\varepsilon>0$

$$
\mathbb{P}\left(\alpha_{n} \geq q g\left(n^{-1}\right) / n\right) \geq \mathbb{P}\left(\Omega_{n}\right) \rightarrow 1
$$

for $n \rightarrow \infty$, with $g\left(n^{-1}\right) \rightarrow \infty$ and $\Omega_{n}$ given in (16). Then (18) and (19) together yield $\mathbb{P}\left(\alpha_{n} \geq 1 / n\right) \rightarrow 1$ for $n \rightarrow \infty$ and therefore the result. 


\subsection{Proof of Corollary 3}

Proof (Corollary 3) Fix $\varepsilon>0$. Denote by $\alpha_{n}$ the output of the discrepancy principle with emergency stop and set

$$
\Omega_{n}:=\left\{\left\|R_{\alpha_{n}} \bar{Y}_{n}-K^{+} \hat{y}\right\| \leq \varepsilon\right\}
$$

It is

$$
\left\|R_{\alpha} \hat{y}-K^{+} \hat{y}\right\| \leq\left\|R_{\alpha} K-I d\right\|\|\hat{x}\| \leq C
$$

for all $\alpha>0$. By the triangle inequality,

$$
\begin{aligned}
\mathbb{E}\left\|R_{\alpha_{n}} \bar{Y}_{n}-K^{+} \hat{y}\right\|^{2} & =2 \mathbb{E}\left\|R_{\alpha_{n}} \bar{Y}_{n}-R_{\alpha_{n}} \hat{y}\right\|^{2}+2 \mathbb{E}\left\|R_{\alpha_{n}} \hat{y}-K^{+} \hat{y}\right\|^{2} \\
& \leq 2 \mathbb{E}\left[\left\|R_{\alpha_{n}}\right\|^{2} \delta_{n}^{\text {true }}\right]+2 C^{2} \leq 2 C_{R} C_{F} \mathbb{E}\left[\delta_{n}^{\text {true }} / \alpha_{n}\right]+2 C^{2} \\
& \leq 2 n C_{R} C_{F} \mathbb{E} \delta_{n}^{\text {true }}{ }^{2}+2 C^{2}=2 C_{R} C_{F} \mathbb{E}\left\|Y_{1}-\hat{y}\right\|^{2}+2 C^{2} \leq C^{\prime},
\end{aligned}
$$

where $C^{\prime}$ does not depend on $n$ and where we used $\alpha_{n} \leq 1$ and (21) in the second step and $\alpha_{n} \geq 1 / n$ in the fourth. By (20) there holds $\left\|R_{\alpha_{n}} \overline{\bar{Y}}_{n}-K^{+} \hat{y}\right\| \chi_{\Omega_{n}} \leq \varepsilon$, so

$$
\begin{aligned}
\mathbb{E}\left\|R_{\alpha_{n}} \bar{Y}_{n}-K^{+} \hat{y}\right\|^{2} & =\mathbb{E}\left[\left\|R_{\alpha_{n}} \bar{Y}_{n}-K^{+} \hat{y}\right\|^{2} \chi_{\Omega_{n}}\right]+\mathbb{E}\left[\left\|R_{\alpha_{n}} \bar{Y}_{n}-K^{+} \hat{y}\right\|^{2} \chi_{\Omega_{n}^{C}}\right] \\
& \leq \varepsilon^{2}+\mathbb{E}\left[\left\|R_{\alpha_{n}} \bar{Y}_{n}-K^{+} \hat{y}\right\|^{2} \chi_{\Omega_{n}^{C}}\right] .
\end{aligned}
$$

We apply Cauchy-Schwartz to the second term

$$
\begin{aligned}
\mathbb{E}\left[\left\|R_{\alpha_{n}} \bar{Y}_{n}-K^{+} \hat{y}\right\|^{2} \chi_{\Omega_{n}^{C}}\right] & \leq \sqrt{\mathbb{E}\left\|R_{\alpha_{n}} \bar{Y}_{n}-K^{+} \hat{y}\right\|^{4} \mathbb{E} \chi_{\Omega_{n}^{C}}^{2}} \\
& =\sqrt{\mathbb{E}\left\|R_{\alpha_{n}} \bar{Y}_{n}-K^{+} \hat{y}\right\|^{4} \mathbb{P}\left(\Omega_{n}^{C}\right)}
\end{aligned}
$$

and we claim that there is a constant $A$ with $\mathbb{E}\left\|R_{\alpha_{n}} \bar{Y}_{n}-K^{+} \hat{y}\right\|^{4} \leq A$ for all $n \in \mathbb{N}$.

$$
\begin{aligned}
\mathbb{E} \| & R_{\alpha_{n}} \bar{Y}_{n}-K^{+} \hat{y} \|^{4} \\
\leq & 4\left(\mathbb{E}\left\|R_{\alpha_{n}} \bar{Y}_{n}-R_{\alpha_{n}} \hat{y}\right\|^{4}+2 \mathbb{E}\left[\left\|R_{\alpha_{n}} \bar{Y}_{n}-R_{\alpha_{n}} \hat{y}\right\|^{2}\left\|R_{\alpha_{n}} \hat{y}-K^{+} \hat{y}\right\|^{2}\right]\right. \\
& \left.+\mathbb{E}\left\|R_{\alpha_{n}} \hat{y}-K^{+} \hat{y}\right\|^{4}\right) \\
\leq & 4\left(\mathbb{E}\left[\left\|R_{\alpha_{n}}\right\|^{4} \delta_{n}^{\text {true }} 4\right]+2 C^{2} \mathbb{E}\left[\left\|R_{\alpha_{n}}\right\|^{2} \delta_{n}^{\text {true }} 2\right]+C^{4}\right) \\
\leq & B\left(\mathbb{E}\left[\delta_{n}^{\text {true }} / \alpha_{n}^{2}\right]+\mathbb{E}\left[\delta_{n}^{\text {true }} 2 / \alpha_{n}\right]+1\right)
\end{aligned}
$$

for some constant $B$, where we used (21) in the second step. First,

$\mathbb{E}\left[\delta_{n}^{\text {true }} 4 / \alpha_{n}^{2}\right]$ 


$$
\begin{aligned}
& \leq n^{2} \mathbb{E}\left\|\bar{Y}_{n}-\hat{y}\right\|^{4}=n^{2} \mathbb{E}\left[\sum_{j, j^{\prime} \geq 1}\left(\bar{Y}_{n}-\hat{y}, u_{j}\right)^{2}\left(\bar{Y}_{n}-\hat{y}, u_{j^{\prime}}\right)^{2}\right] \\
& =\frac{1}{n^{2}}\left(\sum_{j, j^{\prime} \geq 1} \sum_{i, i^{\prime}, l, l^{\prime}=1}^{n} \mathbb{E}\left[\left(Y_{i}-\hat{y}, u_{j}\right)\left(Y_{l}-\hat{y}, u_{j}\right)\left(Y_{i^{\prime}}-\hat{y}, u_{j^{\prime}}\right)\left(Y_{l^{\prime}}-\hat{y}, u_{j^{\prime}}\right)\right]\right) \\
& \leq \frac{1}{n^{2}} \sum_{j, j^{\prime} \geq 1}\left(n \mathbb{E}\left[\left(Y_{1}-\hat{y}, u_{j}\right)^{2}\left(Y_{1}-\hat{y}, u_{j^{\prime}}\right)^{2}\right]\right. \\
& +n^{2} \mathbb{E}\left[\left(Y_{1}-\hat{y}, u_{j}\right)^{2}\right] \mathbb{E}\left[\left(Y_{1}-\hat{y}, u_{j^{\prime}}\right)^{2}\right] \\
& \left.+2 n^{2}\left(\mathbb{E}\left[\left(Y_{1}-\hat{y}, u_{j}\right)\left(Y_{1}-\hat{y}, u_{j^{\prime}}\right)\right]\right)^{2}\right) \\
& \leq \frac{n+2 n^{2}}{n^{2}} \mathbb{E}\left[\sum_{j, j^{\prime} \geq 1}\left(Y_{1}-\hat{y}, u_{j}\right)^{2}\left(Y_{1}-\hat{y}, u_{j^{\prime}}\right)^{2}\right] \\
& +\mathbb{E}\left[\sum_{j \geq 1}\left(Y_{1}-\hat{y}, u_{j}\right)^{2}\right] \mathbb{E}\left[\sum_{j^{\prime} \geq 1}\left(Y_{1}-\hat{y}, u_{j^{\prime}}\right)^{2}\right] \\
& \leq \frac{n+2 n^{2}}{n^{2}} \mathbb{E}\left[\left(\sum_{j \geq 1}\left(Y_{1}-\hat{y}, u_{j}\right)^{2}\right)^{2}\right]+\left(\mathbb{E}\left[\sum_{j \geq 1}\left(Y_{1}-\hat{y}, u_{j}\right)^{2}\right]\right)^{2} \\
& =\frac{n+2 n^{2}}{n^{2}} \mathbb{E}\left\|Y_{1}-\hat{y}\right\|^{4}+\left(\mathbb{E}\left[\left\|Y_{1}-\hat{y}\right\|^{2}\right]\right)^{2} \leq B_{1}
\end{aligned}
$$

for some constant $B_{1}$, where in the fourth step we used that the $Y_{i}$ are i.i.d, that $\mathbb{E}\left(Y_{1}-\hat{y}, u_{j}\right)=\left(\mathbb{E}\left[Y_{1}\right]-\hat{y}, u_{j}\right)=0$ and that $\mathbb{E}[X Y]=\mathbb{E}[X] \mathbb{E}[Y]$ for independent (and integrable) random variables (so the relevant cases are the ones where either all indices $i, i^{\prime}, l, l^{\prime}$ are equal or exactly pairwise two). Then we used Jensen's inequality in the fifth step. Moreover, $\mathbb{E}\left[\delta_{n}^{\text {true }}{ }^{2} / \alpha_{n}\right] \leq n \mathbb{E}\left[\delta_{n}^{\text {true }}{ }^{2}\right]=\mathbb{E}\left\|Y_{1}-\hat{y}\right\|^{2}=B_{2}$, so the claim holds for $A=B\left(B_{1}+B_{2}+1\right)$. By Theorem 3 it holds that $\mathbb{P}\left(\Omega_{n}\right) \rightarrow 1$ for $n \rightarrow \infty$, thus $\mathbb{P}\left(\Omega_{n}^{C}\right) \leq \varepsilon^{4} / A$ for $n$ large enough and

$$
\mathbb{E}\left\|R_{\alpha_{n}} \bar{Y}_{n}-K^{+} \hat{y}\right\|^{2} \leq \varepsilon^{2} \mathbb{E}\left[\chi_{\Omega_{n}}\right]+\sqrt{\mathbb{E}\left\|R_{\alpha_{n}} \bar{Y}_{n}-K^{+} \hat{y}\right\|^{4} \mathbb{P}\left(\Omega_{n}^{C}\right)} \leq 2 \varepsilon^{2}
$$

\section{Numerical demonstration}

We conclude with some numerical results. 


\subsection{Differentiation of binary option prices}

A natural example is given if the data is acquired by a Monte-Carlo simulation, here we consider an example from mathematical finance. The buyer of a binary call option receives after $T$ days a payoff $Q$, if then a certain stock price $S_{T}$ is higher then the strike value $K$. Otherwise he gets nothing. Thus the value $V$ of the binary option depends on the expected evolution of the stock price. We denote by $r$ the riskfree rate, for which we could have invested the buying price of the option until the expiry rate $T$. If we already knew today for sure, that the stock price will hit the strike (insider information), we would pay $V=e^{-r T} Q$ for the binary option $\left(e^{-r T}\right.$ is called discount factor). Otherwise, if we believed that the stock price will hit the strike with probability $p$, we would pay $V=e^{-r T} Q p$. In the Black Scholes model one assumes, that the relative change of the stock price in a short time intervall is normally distributed, that is

$$
S_{t+\delta t}-S_{t} \sim \mathscr{N}\left(\mu \delta t, \sigma^{2} \delta t\right)
$$

Under this assumption one can show that (see [22])

$$
S_{T}=S_{0} e^{s T}
$$

where $S_{0}$ is the initial stock price and $s \sim \mathscr{N}\left(\mu-\sigma^{2} / 2, \sigma^{2} / T\right)$. Under this assumptions one has $V=e^{-r T} Q \Phi(d)$, with

$$
\Phi(x):=\frac{1}{\sqrt{2 \pi}} \int_{-\infty}^{x} e^{-\frac{\xi^{2}}{2}} d \xi, \quad d=\frac{\log \frac{S_{0}}{K}+T\left(\mu-\frac{\sigma^{2}}{2}\right)}{\sigma \sqrt{T}} .
$$

Ultimatively we are interested in the sensitivity of $V$ with respect to the starting stock price $S_{0}$, that is $\partial V\left(S_{0}\right) / \partial S_{0}$. We formulate this as the inverse problem of differentiation. Set $\mathscr{X}=\mathscr{Y}=L^{2}([0,1]=$ and define

$$
\begin{aligned}
K: L^{2}([0,1]) & \rightarrow L^{2}([0,1]) \\
f & \mapsto A f=g: x \mapsto \int_{0}^{x} f(y) d y .
\end{aligned}
$$

Then our true data is $\hat{y}=V=e^{-r T} Q \Phi(d)$. To demonstrate our results we now approximate $V: S_{0} \mapsto e^{-r T} Q p\left(S_{0}\right)$ through a Monte-Carlo approach. That is we generate independent gaussian random variables $Z_{1}, Z_{2}, \ldots$ identically distributed to $s$ and set $Y_{i}:=e^{-r T} Q \chi_{\left\{S_{0} e^{T Z_{i}} \geq K\right\}}$. Then we have $\mathbb{E} Y_{i}=e^{-r T} Q \mathbb{P}\left(S_{0} e^{T Z_{i}}\right)=$ $e^{-r T} Q p\left(S_{0}\right)=V\left(S_{0}\right)$ and $\mathbb{E}\left\|Y_{i}\right\|^{2} \leq e^{-r T} Q<\infty$. We replace $L^{2}([0,1])$ with piecewise continuous linear splines on a homogeneous grid with $m=50$, 000 elements (we can calculate $K g$ exactly for such a spline $g$ ). We use in total $n=10,000$ random variables for each simulation. As parameters we chose $r=0.0001, T=30, K=$ $0.5, Q=1, \mu=0.01, \sigma=0.1$. It is easy to see that $\hat{x}=K^{+} \hat{y} \in \mathscr{X}_{v}$ for all 


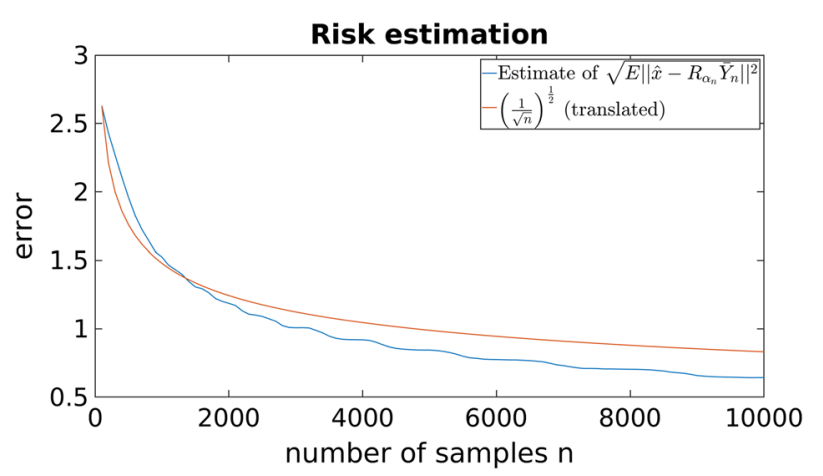

Fig. 1 Estimated risk of a binary option

$v>0$ using the transformation $z(\xi)=0,5 e^{\sqrt{0,3} \xi-0,15}$. Since the qualification of the Tikhonov regularisation is 2 , Theorem 4 gives an error bound which is asymptotically proportional to $(1 / \sqrt{n})^{\frac{1}{2}}$. In Fig. 1 we plot the $L^{2}$ average of 100 simulations of the discrepancy principle together with the (translated) optimal error bound. In this case the emergency stop did not trigger once - this is plausible, since the true solution is very smooth, which yields comparably higher values of the regularisation parameter and also, the error distribution is Gaussian and the problem is only mildly ill-posed. Let us stress that this is only an academic example to demonstrate the possibility of using our new generic approach in the context of Monte Carlo simulations. Explicit solution formulas for standard binary options are well-known, and for more complex financial derivatives with discontinuous payoff profiles (such as autocallables or Cocobonds) one would rather resort to stably differentiable Monte Carlo methods [2] or [14] or use specific regularization methods for numerical differentiation [18].

\subsection{Inverse heat equation}

We consider the toy problem 'heat' from [19]. We chose the discretisation level $m=$ 100 and set $\sigma=0.7$. Under this choice, the last seven singular values (calculated with the function 'csvd') fall below the machine precision of $10^{-16}$. The discretised large systems of linear equations are solved iteratively using the conjugate gradient method ('pcg' from MATLAB) with a tolerance of $10^{-8}$. As a regularisation method we chose Tikhonov regularisation and we compared the a priori choice $\alpha_{n}=1 / \sqrt{n}$, the discrepancy principle (dp) and the discrepancy principle with emergency stop (dp+es), as implemented in Algorithm 1 with $q=0.7$ and estimated sample variance. The unbiased i.i.d measurements fullfill $\sqrt{\mathbb{E}\left\|Y_{i}-\hat{y}\right\|^{2}} \approx 1.16$ and $\mathbb{E}\left\|Y_{i}-\mathbb{E} Y_{i}\right\|^{k}=\infty$ for $k \geq 3$. Concretely, we chose $Y_{i}:=\hat{y}+E_{i}$ with $E_{i}:=U_{i} * Z_{i} * v$, where the $U_{i}$ are independent and uniformly on $[-1 / 2,1 / 2]$ distributed, the $Z_{i}$ are independent Pareto distributed (MATLAB function 'gprnd' with parameters 1/3, 1/2 and 3/2), and $v$ is a uniform permutation of $1,1 / 2^{\frac{3}{4}}, \ldots, 1 / m^{\frac{3}{4}}$. Thus we chose a rather illposed problem together with a heavy-tailed error distribution. We considered three different sample sizes $n=10^{3}, 10^{4}, 10^{5}$ with 200 simulations for each one. The 

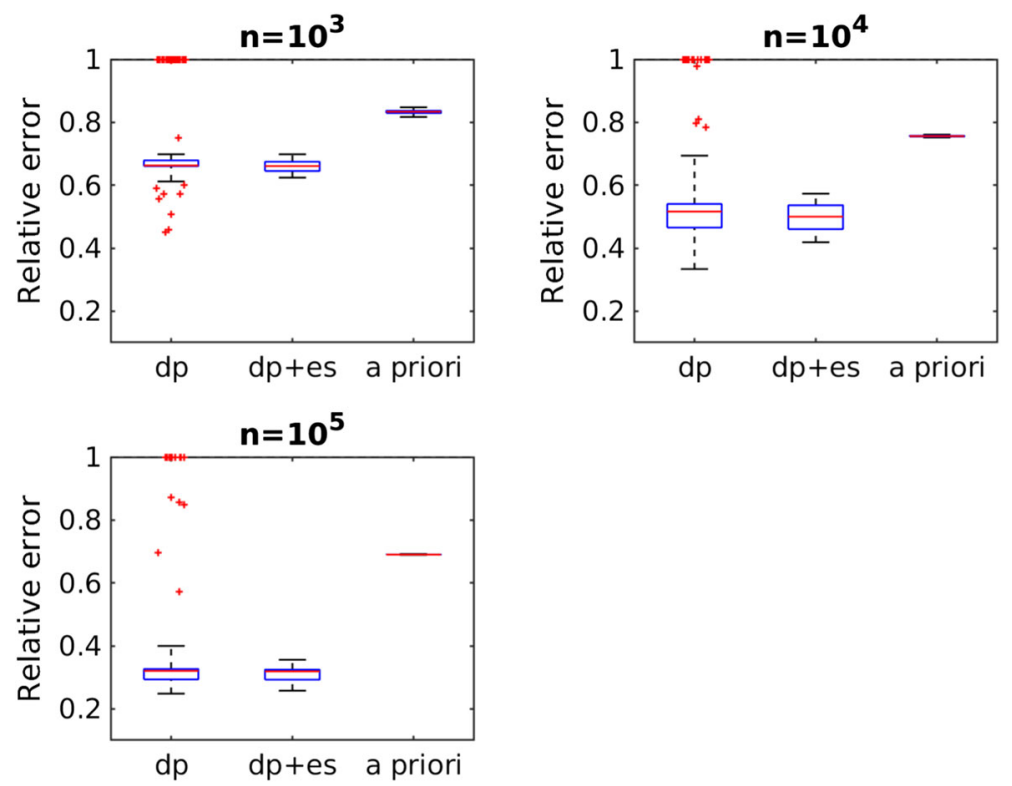

Fig. 2 Comparison of Tikhonov regularisation with discrepancy principle (dp, Algorithm 1), discrepancy principle with emergency stop (dp + es, Algorithm 1 (optional)) and a priori choice for 'heat'. Boxplots of the relative errors $\left\|R_{\alpha_{n}} \bar{Y}_{n}-K^{+} \hat{y}\right\| /\left\|K^{+} \hat{y}\right\|$ for 200 simulations with three different sample sizes

$\begin{array}{lrrr}n=10^{3} & \text { r dp } & \text { dp+es } & \text { a priori } \\ n=10^{4} & 79.45 & 0.66 & 0.83 \\ n=10^{5} & 107.19 & 0.49 & 0.76 \\ & & 0.31 & 0.69\end{array}$

Fig. 3 Estimated relative $L^{2}$ error for 'heat', that is $\sqrt{\sum_{t=1}^{200} e_{i}^{2} / 200}$ where $e_{i}$ is the relative error $\| R_{\alpha_{n}} \bar{Y}_{n}-$ $K^{+} \hat{y}\|\| /\left\|K^{+} \hat{y}\right\|$ of the $i$-th run

results are presented as boxplots in Fig. 2. It is visible, that the results are much more concentrated for a priori regularisation and discrepancy prinicple with emergency stop, indicating the $L^{2}$ convergence (strictly speaking we do not know if the discrepancy principle with emergency stop converges in $L^{2}$, since the additional assumption of Corollary 3 is violated here). Moreover the statistics of the discrepancy principle with and without emergency stop become more similiar with increasing sample size - with the crucial difference, that the outliers as such we denote the red crosses above the blue box, thus the cases where the mehod performed badly) are only present in case of the discrepancy principle without emergency stop, causing non-convergence in $L^{2}$, see Fig. 3. Thus here the discrepancy principle with emergency stop is superior to the discrepancy principle without emergency stop, in particular for large sample sizes. Beside that, the error is falling slower in case of the a priori parameter choice. The number of outliers falls with increasing sample size from 37 for $n=10^{3}$ to 18 for $n=10^{5}$, indicating the (slow) convergence in probability of the discrepancy principle. Note that $\delta_{n}^{\text {true }} / \delta_{n}^{e s t} \approx 1.9$ (in average), if we only consider the runs yielding 
outliers. This illustrates, that the lack of convergence in $L^{2}$ is caused by the occasional underestimation of the data error.

Acknowledgements Open Access funding provided by Projekt DEAL.

Open Access This article is licensed under a Creative Commons Attribution 4.0 International License, which permits use, sharing, adaptation, distribution and reproduction in any medium or format, as long as you give appropriate credit to the original author(s) and the source, provide a link to the Creative Commons licence, and indicate if changes were made. The images or other third party material in this article are included in the article's Creative Commons licence, unless indicated otherwise in a credit line to the material. If material is not included in the article's Creative Commons licence and your intended use is not permitted by statutory regulation or exceeds the permitted use, you will need to obtain permission directly from the copyright holder. To view a copy of this licence, visit http://creativecommons.org/licenses/by/4.0/.

\section{References}

1. Akiyama, K., Alberdi, A., Alef, W., Asada, K., Azulay, R., Baczko, A.K., Ball, D., Baloković, M., Barrett, J., Bintley, D., et al.: First M87 event horizon telescope results. III. Data processing and calibration. Astrophys. J. Lett. 875(1), L3 (2019)

2. Alm, T., Harrach, B., Harrach, D., Keller, M.: A Monte Carlo pricing algorithm for autocallables that allows for stable differentiation. J. Comput. Finance 17(1), 43-70 (2013)

3. Bakushinski1, A.: Remarks on the choice of regularization parameter from quasioptimality and relation tests. Zh. Vychisl. Mat. i Mat. Fiz. 24(8), 1258-1259 (1984)

4. Bauer, F., Reiß, M.: Regularization independent of the noise level: an analysis of quasi-optimality. Inverse Probl. 24(5), 055009 (2008)

5. Becker, S.: Regularization of statistical inverse problems and the Bakushinskiur veto. Inverse Probl. 27(11), 115010 (2011)

6. Bissantz, N., Hohage, T., Munk, A., Ruymgaart, F.: Convergence rates of general regularization methods for statistical inverse problems and applications. SIAM J. Numer. Anal. 45(6), 2610-2636 (2007)

7. Blanchard, G., Hoffmann, M., Reiß, M.: Optimal adaptation for early stopping in statistical inverse problems. SIAM/ASA J. Uncertain. Quantif. 6(3), 1043-1075 (2018)

8. Blanchard, G., Mathé, P.: Discrepancy principle for statistical inverse problems with application to conjugate gradient iteration. Inverse Probl. 28(11), 115011 (2012)

9. Buades, T., Lou, Y., Morel, J.M., Tang, Z.: A note on multi-image denoising. In: 2009 International Workshop on Local and Non-local Approximation in Image Processing, pp. 1-15. IEEE (2009)

10. Cavalier, L.: Inverse problems in statistics. In: Alquier, P., Gautier, E., Stoltz, G. (eds.) Inverse Problems and High-Dimensional Estimation, pp. 3-96. Springer, Berlin (2011)

11. Cavalier, L., Golubev, Y., et al.: Risk hull method and regularization by projections of ill-posed inverse problems. Ann. Stat. 34(4), 1653-1677 (2006)

12. Engl, H.W., Hanke, M., Neubauer, A.: Regularization of Inverse Problems, vol. 375. Springer, Berlin (1996)

13. Garcia, E.S., Sandwell, D.T., Smith, W.H.: Retracking CryoSat-2, Envisat and Jason-1 radar altimetry waveforms for improved gravity field recovery. Geophys. J. Int. 196(3), 1402-1422 (2014)

14. Gerstner, T., Harrach, B., Roth, D.: Monte Carlo pathwise sensitivities for barrier options. J. Comput. Finance, accepted for publication (2018)

15. Gerth, D., Hofinger, A., Ramlau, R.: On the lifting of deterministic convergence rates for inverse problems with stochastic noise. Inverse Probl. Imaging 11(4), 663-687 (2017)

16. Ghosal, S., Van der Vaart, A.: Fundamentals of Nonparametric Bayesian Inference, vol. 44. Cambridge University Press, Cambridge (2017)

17. Giné, E., Nickl, R.: Mathematical Foundations of Infinite-Dimensional Statistical Models, vol. 40. Cambridge University Press, Cambridge (2016)

18. Hanke, M., Scherzer, O.: Inverse problems light: numerical differentiation. Am. Math. Mon. 108(6), 512-521 (2001)

19. Hansen, P.C.: Discrete Inverse Problems: Insight and Algorithms, vol. 7. SIAM, Philadelphia (2010) 
20. Hassan, U., Anwar, M.S.: Reducing noise by repetition: introduction to signal averaging. Eur. J. Phys. 31(3), 453 (2010)

21. Hofinger, A.: Ill-Posed Problems: Extending the Deterministic Theory to a Stochastic Setup. Trauner, New York (2006)

22. Hull, J.C., Basu, S.: Options, Futures, and Other Derivatives. Pearson Education India, Chennai (2016)

23. Kaipio, J., Somersalo, E.: Statistical and Computational Inverse Problems, vol. 160. Springer, Berlin (2006)

24. Ledoux, M., Talagrand, M.: Probability in Banach Spaces: Isoperimetry and Processes, vol. 23. Springer, Berlin (1991)

25. Lu, S., Mathé, P.: Discrepancy based model selection in statistical inverse problems. J. Complex. 30(3), 290-308 (2014)

26. Lucka, F., Proksch, K., Brune, C., Bissantz, N., Burger, M., Dette, H., Wübbeling, F.: Risk estimators for choosing regularization parameters in ill-posed problems-properties and limitations. Inverse Probl. Imaging 12(5), 1121-1155 (2018)

27. Lyons, R.G.: Understanding Digital Signal Processing, 3/E. Pearson Education India, Chennai (2004)

28. Mackay, C.D., Baldwin, J., Law, N., Warner, P.: High-resolution imaging in the visible from the ground without adaptive optics: new techniques and results. In: Ground-Based Instrumentation for Astronomy, vol. 5492, pp. 128-136. International Society for Optics and Photonics (2004)

29. Mathé, P., Pereverzev, S.V.: Geometry of linear ill-posed problems in variable Hilbert scales. Inverse Probl. 19(3), 789 (2003)

30. Morozov, V.A.: The error principle in the solution of operational equations by the regularization method. Zhurnal Vychislitel'noi Matematiki i Matematicheskoi Fiziki 8(2), 295-309 (1968)

31. Nakamura, G., Potthast, R.: Inverse Modeling, pp. 2053-2563. IOP Publishing, Bristol (2015). https:// doi.org/10.1088/978-0-7503-1218-9

32. Rieder, A.: Keine Probleme mit inversen Problemen: eine Einführung in ihre stabile Lösung. Springer, Berlin (2013)

33. Tikhonov, A., Arsenin, V.Y.: Methods for Solving Ill-Posed Problems. Wiley, New York (1977)

34. Wahba, G.: Practical approximate solutions to linear operator equations when the data are noisy. SIAM J. Numer. Anal. 14(4), 651-667 (1977)

35. Werner, F.: Adaptivity and Oracle inequalities in linear statistical inverse problems: a (numerical) survey. In: Hofmann, B., Leitão, A., Zubelli, J.P. (eds.) New Trends in Parameter Identification for Mathematical Models, pp. 291-316. Springer, Berlin (2018)

Publisher's Note Springer Nature remains neutral with regard to jurisdictional claims in published maps and institutional affiliations. 